\title{
On the Asymptotic Exactness of the Bogoliubov Approximation for Many Boson Systems*
}

\author{
J. GINIBRE** \\ Department of Physics, New York University
}

Received November 15, 1967

\begin{abstract}
The Bogoliubov approximation for many boson systems consists in replacing the field operators $a_{0}$ and $a_{0}^{+}$by $c$-numbers, to be determined by an extremum condition. Here we formulate the approximation in terms of coherent states of the condensed particles, and prove that for reasonable interactions it gives the exact values of the thermodynamical functions in the infinite volume limit.
\end{abstract}

\section{Introduction}

The standard procedure to describe a many boson system in thermal equilibrium, exhibiting Bose Einstein condensation, is the Bogoliubov approximation (hereafter called B.A.), which was proposed as early as 1947, [1]. One takes advantage of the macroscopic occupation of the zero momentum one particle state to replace the corresponding creation and annihilation operators $a_{0}$ and $a_{0}^{+}$by $c$-numbers. The replacement is supposed to be exact in the infinite volume limit. The validity of the B.A., however, has not been rigorously proved, and has even been questioned [2]. Arguments to support it have been given in the case of the ground state energy, with special types of interactions $[3,4]$. In the present paper, we want to make a first step towards a general proof of its asymptotic exactness in the limit of infinite systems.

In order to show how the present work fits into the general scheme, we first review the latter briefly and qualitatively, following BogoLIUвov [5] and HAAG [6]. We consider a many boson system in equilibrium, at a given non-zero temperature $T(\beta=1 / k T)$, a given chemical potential $\mu$, and with given interactions. The system is described in the grand canonical formalism (that this is at all possible will be shown in Sec. 1). We are interested in the infinite volume limit, with possible occurrence of Bose-Einstein condensation.

* Research supported in part by the National Science Foundation.

** Permanent address: Laboratoire de Physique Théorique, Faculté des Sciences, Orsay (France). 
(1) It has been argued by HAAG [6] in the case of the BCS model that if one describes the state of the infinite system in an irreducible representation of the algebra generated by the field operators (namely the algebra of the canonical anticommutation relations in the case of the BCS model), then the space average of any local quantity should be represented by a constant. In the case of a boson system, the same argument applies to the operators $V^{-1 / 2} a_{0}$ and $V^{-1 / 2} a_{0}^{+}$, which are the space averages of the field operators $a(x)$ and $a^{+}(x)$ themselves. Therefore, in an irreducible representation of the basic algebra (in this case the algebra of the canonical commutation relations), these operators should also be represented by $c$-numbers. This is the basis for the B.A. Notice that the occurrence of Bose-Einstein condensation is not required in that argument.

(2) We now consider a finite system. The preceding argument suggests replacing $a_{0}$ and $a_{0}^{+}$by $c$-numbers $C$ and $\bar{C}$. In the presence of condensation, $C$ should turn out to be of the order $V^{1 / 2}$. As will be seen in Section 3, this replacement can be made in several ways, two of which are of special interest. The first consists in making the replacement in the statistical operator $W=\exp (-\beta H)$, where $H$ is the hamiltonian. $H$ contains a term $-\mu N$, since we are working in the grand canonical formalism. One then obtains an approximate operator $W_{0}(C)$ and an approximate expression for the grand canonical pressure

$$
\beta p_{0}(C)=V^{-1} \log \left[\operatorname{Tr} W_{0}(C)\right] .
$$

The second way consists in performing the replacement in $H$ itself, as was proposed originally by ВоGоцтво [1]. It gives an approximate hamiltonian $H_{0}(C)$ and an approximate pressure:

$$
\beta p_{0}^{\prime}(C)=V^{-1} \log \operatorname{Tr} \exp \left[-\beta H_{0}(C)\right] .
$$

In both cases, the B.A. has to be supplemented with some prescription to determine $C$.

(3) We shall then prove that:

where

$$
p_{0}^{\prime}(C) \leqq p_{0}(C) \leqq p
$$

$$
\beta p=V^{-1} \log \operatorname{Tr} W .
$$

One should therefore determine $C$ in both cases by the condition that the approximate pressure be maximal. Furthermore, as a justification of the B.A., we shall prove that both $\operatorname{Sup}_{C} p_{0}(C)$ and $\operatorname{Sup}_{C} p_{0}^{\prime}(C)$ tend to the same limit $\tilde{p}$ as $p$ when the system becomes infinite.

(4) We return to the maximum problem. In the second case (B.A. in $H$ ), it leads immediately to an equation for $C$, which may be called the 
condensate equation [5]. This equation stands in close analogy with the gap equation of the B.C.S. model. It shares with the latter the property that it always has the trivial solution $C=0$.

(5) In the case where the maximum problem leads to a non-zero value of $C$, it determines only $|C|$, but not the argument of $C$. This corresponds to the case where condensation actually occurs. The state of the system obtained by the standard limiting procedure starting from $W$ is then expected to exhibit a degeneracy under gauge transformations and to be decomposable as a convex combination of non-gauge invariant states. A possible way to obtain these non-invariant states directly is to add to the hamiltonian an infinitesimal source term $-\sqrt{V}\left(\nu a_{0}^{+}+\bar{\nu} a_{0}\right)$, where $\nu$ is a complex parameter which eventually goes to zero after the infinite volume limit has been taken.

(6) The same procedure as described under (2) and (3) can be repeated with this term added to the hamiltonian. The inequality (3) still holds, as well as the asymptotic equality of the various pressures. The maximum problem then determines the argument of $C$ as being equal to that of $\nu$. The source term plays the same role as an infinitesimal magnetic field orienting a ferromagnet.

In the present paper, as a first step toward proving the asymptotic exactness of the B.A., we shall prove the statements relative to the thermodynamical functions, listed under (3), both for $v=0$ and $v \neq 0$.

More interesting, would be to prove the results concerning the state of the system, as listed under (5). This is however a much more difficult problem. The existence of the infinite volume limit itself has been established only in very restrictive cases, where fugacity expansions could be used, and therefore in a range of the parameters where condensation certainly does not occur. We shall therefore not consider this problem, except for a few trivial remarks.

Before going to the details of the proof, we would like to insist on the following point. The asymptotic exactness of the B.A. is a general property which holds irrespective of the dimension of the space, the values of $\beta$ and $\mu$, and, within reasonable limits, of the details of the interactions. It does not depend on the presence or absence of condensation. Whether there is condensation or not is a more delicate question (somewhat analogous to that of finding non-trivial solutions to the gap equation of the BCS model), which requires separate investigation and can be formulated within the B.A. Needless to say, however, the B.A. has practical interest only if condensation occurs.

This paper is divided as follows. In Section 1, we describe the conditions imposed on the interactions and deduce from them some useful bounds for the pressure. We then state the relevant theorems $[7,8]$ on 
the existence of the infinite volume limit for the pressure, both for $\nu=0$ and $\nu \neq 0$, as well as some properties of the limiting functions. In Section 2, we state some operator relations $[5,9]$ which will be used in Section 5, and some of their consequences. Both Sections 1 and 2 are rather technical and can be skipped by the reader who wants to get quickly to the main point. In Section 3, we give a convenient definition of the B.A. and apply it to the statistical operator $W$. We then prove the results relative to $p_{0}(C)$. In Section 4 , we make a few remarks on the state of the system, but do not attempt to take the infinite volume limit. In Section 5, we apply the B.A. to the hamiltonian, and prove the results relative to $p_{0}^{\prime}(C)$. Section 6 summarizes the results, and contains some additional remarks.

\section{Conditions on the Potential and Infinite Volume Limit}

We consider a system of identical bosons in $v_{0}$ dimensional euclidean space, enclosed in a box $\Lambda$ of volume $V$, which is a bounded open connected subset of $R^{\nu_{0}}$. We take the common mass of the particles equal to one, and $\hbar=1$. Let $\lambda=(2 \pi \beta)^{1 / 2}$ be the thermal wavelength. The particles interact through a two body potential $\phi$ satisfying the following conditions $[7,8]$ :

(A) $\phi$ is a real even function of the difference of the positions of the two interacting particles. $\phi$ may have a hard core. $\phi$ is locally square integrable on the complement of the closure of the hard core if there is any, and on the complement of the origin, if not.

(B) $\phi$ is superstable, by which we mean that there exist two real constants $B \geqq 0$ and $A_{1}>0$ such that, for any family $\left(x_{1}, \ldots, x_{n}\right)$ of $n$ points such that the associated hard cores do not overlap, the following inequality holds:

where

$$
\sum_{i<j} \phi\left(x_{i}-x_{j}\right) \equiv U\left(x_{1}, \ldots, x_{n}\right) \geqq-n B+A_{1} n^{2} \xi^{-v_{0}}
$$

$$
\xi=\max \left|x_{i}-x_{j}\right| .
$$

Condition (B) is slightly stronger than the usual stability condition $[8,10]$ under which the existence of the infinite volume limit for the thermodynamical functions is proved. However, almost all known interesting examples of stable potentials are of the form $\phi=\phi_{1}+\phi_{2}$ where $\phi_{1}$ is a positive function and $\phi_{2}$ a positive type function $[7,11,12]$. For such potentials, one can show by a slight modification of the arguments in [11] that if $\phi_{2} \neq 0$, then condition (B) is satisfied. Therefore (B) is reasonable. An important exception is the case of free particules, where $\phi=0$ is stable, but not superstable. From the point of view of condition (B), the free case is a pathological limiting case. This is reflected 
most clearly by the inadequacy of the grand canonical formalism to describe free bosons undergoing condensation: the chemical potential remains constant as soon as condensation occurs. On the contrary, the same formalism will be seen to be adequate for superstable bosons, regardless of possible condensation.

It follows immediately from (B) that for any box $\Lambda$, there exists a constant $A>0$ such that for any family $\left(x_{1}, \ldots, x_{n}\right)$ of points in $\Lambda$ :

$$
U\left(x_{1}, \ldots, x_{n}\right) \geqq-n B+A n^{2} V^{-1} \text {. }
$$

$A$ is volume independent for a given shape, but may be shape dependent. Therefore, when considering the infinite volume limit, we shall restrict our attention to families of boxes $\Lambda$ that satisfy the following condition:

(F) There exists a constant $\alpha>1$ such that for any $\Lambda$ in the family:

$$
V^{-1} l^{\nu_{0}} \leqq \alpha
$$

where $l$ is the diameter of $\Lambda$, i.e. the maximum distance of two points of $\Lambda$.

(F) implies that for all $\Lambda$ in the family, (1.3) holds with the same constant $A=A_{1} \alpha^{-1}$.

We need a third condition on $\phi$ :

(C) $\phi$ is weakly tempered [8], i.e. there exist real constants $R>0$, $\phi_{0} \geqq 0$ and $\varepsilon>0$ such that for $|\overrightarrow{\mathrm{r}}| \equiv r \geqq R$ :

$$
\phi(\overrightarrow{\mathrm{r}}) \leqq \phi_{0} r^{-\left(v_{0}+\varepsilon\right)} .
$$

A fourth condition will be needed and introduced in Section 5 .

We now deduce from (B) upper bounds for the grand canonical pressure and density. Let $\mathscr{H}$ be the Fock space of the system, i.e. the symmetric tensor algebra constructed on the one particle space $L^{2}(\Lambda)$. The hamiltonian of the system is:

$$
H=T-\mu N-V^{1 / 2}\left(\bar{\nu} a_{0}+\nu a_{0}^{+}\right)+U
$$

where $T$ is the kinetic energy, $N$ the particle number operator, $U$ the potential energy as defined in (1.1), $v$ any complex number, and

$$
a_{0}=V^{-1 / 2} \int_{\Lambda} a(x) d x,
$$

where $a(x)$ is the boson field operator. - means complex conjugation. $H$ can be defined as a self-adjoint operator by the extension method of FrIEdRICHS [13], using arguments similar to those in ref. [7], hereafter referred to as $R$. We shall need some lemmas from $R$, as well as the following results:

Lemma 1. Let $X$ and $U$ be a positive hermitian and a unitary matrix respectively. Then:

$$
\begin{aligned}
\left|\operatorname{Tr}(X U)^{2^{p+1}}\right| & \leqq \operatorname{Tr}\left(X U X U^{+}\right)^{2^{p}}, \\
\operatorname{Tr}\left(X U X U^{+}\right)^{2^{p+1}} & \leqq \operatorname{Tr}\left(X^{2} U X^{2} U^{+}\right)^{2^{p}}, \\
\left|\operatorname{Tr}(X U)^{2^{p}}\right| & \leqq \operatorname{Tr} X^{2^{p}} .
\end{aligned}
$$


The proof will be omitted. It rests on the use of the inequality $\left|\operatorname{Tr} A^{2}\right| \leqq \operatorname{Tr} A^{+} A$, and on the cyclic property of the trace operation. (1.10) is a special case of a result due to WEYL [14].

Lemma 2. Let $F$ and $G$ be hermitian matrices. Then:

$$
|\operatorname{Tr} \exp (F+i G)| \leqq \operatorname{Tr} \exp F .
$$

Proof. The lemma follows immediately from the relation [15]:

$$
\exp (F+i G)=\lim _{p \rightarrow \infty}\left[\exp \left(2^{-p} F^{\prime}\right) \exp \left(i 2^{-p} G\right)\right]^{2^{p}}
$$

and from (1.10) with $X=\exp \left(2^{-p} F\right)$ and $U=\exp \left(i 2^{-p} G\right)$.

Lemma 3. Let $F$ and $G$ be symmetric operators in $\mathscr{H}$ with common dense domain. Let $t_{1}$ and $t_{2}$ be real numbers, $t_{1}<t_{2}$. Let $F+t G$ be semi-bounded from below for $t=t_{1}$ and $t=t_{2}$, and therefore also for $t_{1} \leqq t \leqq t_{2}$. Let $(F+t G)_{f}$ be its Friedrichs extension and suppose that $\operatorname{Tr} \exp \left[-\left(F+t_{i} G\right)_{f}\right]$ is finite for $i=1,2$. Then:

(I) $\operatorname{Tr} \exp \left[-(F+t G)_{f}\right]$ exists and is a logarithmically convex function of $t$ for $t$ real, $t_{1} \leqq t \leqq t_{2}$.

(II) This function can be continued to an analytic function $f(t)$ in the domain $\mathscr{D}$ defined by

$$
t_{1}<\operatorname{Re} t<t_{2} .
$$

$f$ is uniformly bounded in $\mathscr{D}$. For $t$ real, its derivative is given by:

$$
\frac{d f}{d t}=-\operatorname{Tr} G \exp \left[-(F+t G)_{f}\right] .
$$

Proof. Part I is already contained in Lemmas $\mathrm{R} 2$ and $\mathrm{R} 4$. It will be proved here in a slightly different way.

Let $P$ be a finite dimensional projection operator and let:

$$
f_{P}(t)=\operatorname{Tr} P \exp [-P(F+t G)] .
$$

Then $f_{P}$ is an entire function of $t$. As a particular case of a subsequent result, (2.11), which obviously applies to the finite dimensional case, we have: $d^{2} \log f_{P} / d t^{2} \geqq 0$, so that $\log f_{P}$ is convex on the real $t$ axis. From Lemma R2, Lemma 2, and logarithmic convexity for real $t$, it follows that $f_{P}(t)$ is bounded uniformly for $t_{1} \leqq \operatorname{Re} t \leqq t_{2}$ by:

$$
\left|f_{P}(t)\right| \leqq \max _{i=1,2} \operatorname{Tr} \exp \left[-\left(F+t_{i} G\right)_{f}\right] .
$$

For $t$ real, it follows from Lemma $\mathrm{R} 1$ that $f_{P}(t)$ is an increasing function of $P$. Let $P_{n}$ be a sequence of projectors which increases to the unit operator, and let $f_{P_{n}}=f_{n}$. Then for $t_{1} \leqq t \leqq t_{2}, f_{n}$ converges to a limit as $n \rightarrow \infty$. It then follows from Vitali's theorem [16] that as $n \rightarrow \infty, f_{n}(t)$ converges to an analytic function $f(t)$ in the domain $\mathscr{D}$. The convergence is uniform on the compact sets in $\mathscr{D}$. It follows from (1.16) that $f(t)$ is uniformly bounded in $\mathscr{D}$. For $t$ real, $t_{1} \leqq t \leqq t_{2}$, it follows from Lemma R2 
that the limiting function is $\operatorname{Tr} \exp \left[-(F+t G)_{f}\right]$, which is therefore a bounded logarithmically convex function of $t$ in the same interval.

The derivative of $f_{P}(t)$ is

$$
\frac{d f_{P}}{d t}=\operatorname{Tr} P G P \exp [-P(F+t G)]
$$

From Vitali's theorem, it converges to $d f / d t$ for $t \in \mathscr{D}$. For $t$ real, if we take for $P_{n}$ an increasing sequence of spectral projections of $(F+t G)_{f}$, then $f_{n}^{\prime}(t)$ converges to $\operatorname{Tr} G \exp \left[-(F+t G)_{f}\right]$, which therefore exists and is equal to $d f / d t$. This concludes the proof of Lemma 3.

We now come back to our problem. Let:

$$
\begin{aligned}
W & =\exp (-\beta H), \\
Z & =\operatorname{Tr} W, \\
\beta p & =V^{-1} \log Z .
\end{aligned}
$$

$\beta$ will be considered a parameter throughout. $p$ is a function of $\mu, \nu$, and depends also on $\Lambda$. Whenever a family of boxes is considered, it is understood that they satisfy (F). We then prove the following result.

Lemma 4. (I) $p$ is bounded uniformly with respect to $V$ by a positive function $\hat{p}(\mu, v)$, independent of $V$, for $V \geqq v>0$.

(II) For fixed $\nu, p$ is increasing and convex in $\mu$ on the real axis. It is analytic in $\mu$ in a neighborhood of the real axis.

(III) We fix $\mu$. Let $\nu=r e^{i \omega}$ with $r$ and $\omega$ real numbers. For fixed $r, p$ is independent of $\omega . p$ is real analytic in $r$, convex and increasing for $r \geqq 0$ ( $p$ is an increasing function of $|v|$ ).

(IV) It follows from II, III, that $\hat{p}$ can be taken as an increasing convex function of $\mu$, and an increasing convex function of $|\nu|$.

Proof. We prove (I) in several steps:

(1) $\nu=0, \mu<-B$.

It follows from (1.3) and Lemma R3 that:

$$
Z \leqq \operatorname{Tr}[\exp -\beta(T-(\mu+B) N)]
$$

The RHS is the free particle grand partition function at chemical potential $\mu+B<0$. The corresponding $p$ is bounded by its infinite volume limit [17]

where

$$
\beta p \leqq \lambda^{-v_{0}} g_{1+v_{0} / 2}\left(e^{\beta(\mu+B)}\right)
$$

In particular

$$
g_{\alpha}(z)=\sum_{l>1} z^{\imath} / l^{\alpha}
$$

$$
\beta p \leqq-\lambda^{-\nu_{0}} \log \left(1-e^{\beta(\mu+B)}\right) .
$$

(2) $\nu=0, \mu$ arbitrary. 
Let $H_{n}=T_{n}+U_{n}$ be the component of $T+U$ in the $n$ particle subspace of $\mathscr{H}$. Let

$$
Q_{n}=\operatorname{Tr} \exp \left(-\beta H_{n}\right)
$$

It follows from (1.3) and Lemma R3 that:

where:

$$
Q_{n} \leqq \exp \left[\beta\left(n B-n^{2} A V^{-1}\right)\right] Q_{n}^{(0)}
$$

$$
Q_{n}^{(0)}=\operatorname{Tr} \exp \left(-\beta T_{n}\right)
$$

Using the expression (1.22) for the grand canonical pressure of free bosons, we obtain, with $\varrho=n V^{-1}$ :

$$
\begin{aligned}
V^{-1} \log Q_{n}^{(0)} & \leqq \min _{0 \leqq z<1}\left(-\varrho \log z+\lambda^{-v_{0}} g_{1+v_{0} / 2}(z)\right) \\
& \leqq \min _{0 \leqq z<1}\left(-\varrho \log z-\lambda^{-v_{0}} \log (1-z)\right) \\
& =\varrho \log \left(\varrho \lambda^{\nu_{0}}+1\right) / \varrho \lambda^{v_{0}}+\lambda^{-v_{0}} \log \left(1+\varrho \lambda^{v_{0}}\right) \\
& \leqq \lambda^{-v_{0}}+\varrho .
\end{aligned}
$$

Therefore

$$
Q_{n}^{(0)} \leqq \exp \left(V \lambda^{-v_{0}}+n\right)
$$

Substituting $(1.26,32)$ into:

$$
Z=\sum_{n} e^{n \beta \mu} Q_{n}
$$

we obtain:

$$
\begin{aligned}
Z & \leqq \sum_{n} \exp \left\{V \lambda^{-v_{0}}+n[1+\beta(\mu+B)]-A \beta n^{2} V^{-1}\right\} \\
& \leqq \exp \left\{V\left(\lambda^{-v_{0}}+[1+\beta(\mu+B)]^{2} / 4 A \beta\right)\right\}\left\{1+(2 \pi V / A \beta)^{1 / 2}\right\} .
\end{aligned}
$$

Therefore

$$
\beta p \leqq \lambda^{-v_{0}}+[1+\beta(\mu+B)]^{2} / 4 A \beta+\varepsilon(V)
$$

where $\varepsilon(V)$ is bounded uniformly in $V$ for $V \geqq v>0$. (1.24) and (1.36) prove part (I) of Lemma 4 for $v=0$. Anticipating (II), we apply (IV) and take $\hat{p}(\mu, 0)$ to be the convex envelope of the functions defined by (1.24) and (1.36) (see Fig. 1).

(3) $\nu \neq 0, \mu$ arbitrary.

It follows from $a_{0}^{+} a_{0} \leqq N$ that:

$$
V^{1 / 2}\left(\bar{\nu} a_{0}+\nu a_{0}^{+}\right) \geqq-2|\nu|(N V)^{1 / 2} \geqq-|\nu|(N+V) .
$$

Then, from Lemma R3:

$$
p(\mu, \nu) \leqq|\nu|+p(\mu+|\nu|, 0) .
$$

(I) follows for arbitrary $v$, with:

$$
\hat{p}(\mu, \nu)=|\nu|+\hat{p}(\mu+|\nu|, 0) .
$$


We now come to (II). The properties stated are well known for $v=0[7,8]$. More generally, it follows from Lemma 3 that $Z$ is an entire function of $\mu$, and from III that $Z$ cannot be zero on the real $\mu$ axis. Therefore $p$ is analytic in $\mu$ in a neighborhood of the real axis. Convexity of $p$ follows from Lemma 3. That $p$ is increasing follows from the relation:

$$
\frac{d p}{d \mu}=V^{-1}\langle N\rangle \text {. }
$$

We now come to III. Independence of $p$ on $\omega$ follows from gauge invariance:

$$
Z=\operatorname{Tr} W=\operatorname{Tr} e^{i \alpha N} W e^{-i \alpha N} .
$$

Real analyticity in $r$ and convexity follow from Lemma 3. Monotony will be proved in Section 2. This ends the proof of Lemma 4.

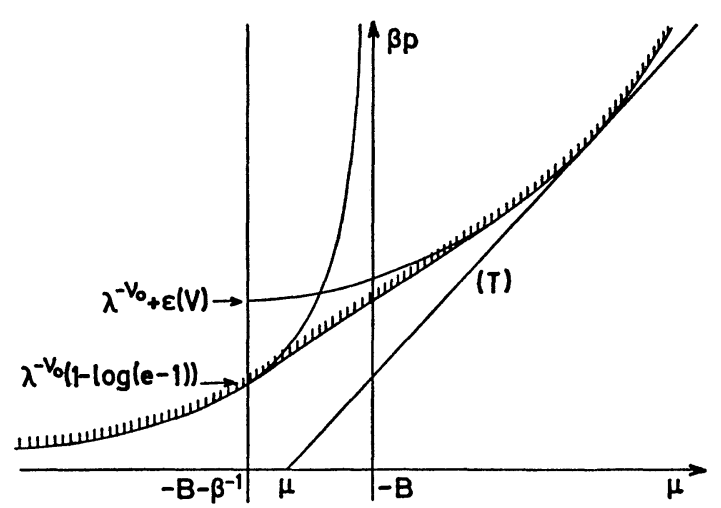

Fig. 1. Upper bounds for $p$ : we have represented the bounds (1.24), (1.36) and their convex envelope. $\hat{\varrho}(\mu)=$ slope of $(T)$.

From the convexity of $p$ as a function of $\mu$ and from the preceding bounds, it follows that the density $\varrho=d p / d \mu$ is bounded for any $\mu$ by the slope of the tangent to the graph of $\hat{p}(\mu)$ from the point $(\mu, 0)$ (see Fig. 1). Let $\hat{\varrho}(\mu, v)$ or simply $\hat{\varrho}$ be this bound. In particular, for $\mu$ sufficiently large, it follows from (1.36) that:

$$
\hat{\varrho}(\mu, 0) \leqq[1+\beta(\mu+B)] / A \beta+2 \lambda^{-v_{0}}[1+\beta(\mu+B)]^{-1} .
$$

This bound behaves as $\mu / A$ for $\mu \rightarrow+\infty$.

We now turn to the problem of the infinite volume limit for the pressure, and quote the relevant result from ref. [8].

Theorem 1. Let $\phi$ satisfy (A), (B) and (C). Let $p$ be defined by (1.20) and let $\Lambda_{j}$ be a sequence of boxes with volumes $V_{j}$ increasing to infinity, satisfying condition ( $\mathrm{F})$ and the uniform regularity condition of ref. [8]. 
Then, as $j \rightarrow \infty, p$ tends to a well defined limit $\tilde{p}$. For fixed $\nu, \tilde{p}$ is a convex, continuous and increasing function of $\mu$. For fixed $\mu$, it depends on $\nu$ only through $r=|v| . \tilde{p}$ is a convex, continuous and increasing function of $r$.

Proof. The proof goes as in [8], with two additional remarks.

(1) Condition (B) supplemented with (F) allows the use of the grand canonical formalism from the beginning.

(2) If $\nu \neq 0$ the additional term

$$
-\int_{\Lambda} d x\left[\bar{v} a(x)+v a^{+}(x)\right]
$$

is an additive function of the box, and therefore does not cause any difficulty.

The properties of the limiting $\tilde{p}$ as a function of $\mu, \nu$ follow from the existence of the limit and from Lemma 4.

\section{Some Operator Relations}

We list here for later use some operator relations due to Bogourubov [5] (see also ref. [9]). Let $H$ be defined as in Section 1. Let $A$ and $B$ be operators in $\mathscr{H}$, such that $|A|$ and $|B|$ are bounded by some power of $N$. We define the following scalar product:

$$
(A, B)=\beta^{-1} Z^{-1} \int_{0}^{\beta} d \lambda \operatorname{Tr}\left(e^{-(\beta-\lambda) B} A^{+} e^{-\lambda B} B\right) .
$$

It is positive semi-definite. Besides the usual relation $(A, B)=(\overline{B, A})$, it also satisfies the symmetry property:

$$
(A, B)=\left(B^{+}, A^{+}\right)
$$

where + means the adjoint operator. Furthermore:

$$
(\mathbf{1}, A)=\langle A\rangle \text {. }
$$

If $B$ is of the form $B=[C, H]$, it is easily seen that:

$$
(A, B)=\beta^{-1}\left\langle\left[C, A^{+}\right]\right\rangle \text {. }
$$

In particular, for any operator $A$,

$$
\left\langle\left[A,\left[H, A^{+}\right]\right]\right\rangle=\beta([A, H],[A, H]) \geqq 0 .
$$

We now compare $(A, A)$ with the anticommutator of $A$ and $A^{+}$. Introducing a spectral decomposition of $H$ we obtain:

$$
(A, A)=Z^{-1} \sum_{m, n}\left|A_{m n}\right|^{2}\left(e^{-\beta E_{m}}-e^{-\beta E_{n}}\right) / \beta\left(E_{n}-E_{m}\right) .
$$

From the elementary inequality:

$\frac{1}{2}\left(e^{x}+e^{y}\right)-\frac{1}{2}\left|e^{x}-e^{y}\right|=\min \left(e^{x}, e^{y}\right) \leqq \frac{e^{x}-e^{y}}{x-y} \leqq \frac{1}{2}\left(e^{x}+e^{y}\right)$ 
we deduce:

$$
\begin{aligned}
(A, A) \leqq & \frac{1}{2}\left\langle\left[A, A^{+}\right]_{+}\right\rangle \leqq(A, A)+\frac{1}{2} Z^{-1} \\
& \cdot \sum_{m, n}\left|A_{m n}\right|^{2}\left|e^{-\beta E_{n}}-e^{-\beta E_{m}}\right| .
\end{aligned}
$$

Schwarz' inequality gives for the last term the following bound:

$$
\begin{aligned}
& \frac{1}{2}\left\{Z^{-1} \sum_{m, n}\left|A_{m n}\right|^{2} \frac{e^{-\beta E_{n}}-e^{-\beta E_{m}}}{\beta\left(E_{m}-E_{n}\right)}\right\}^{1 / 2} \\
& \cdot\left\{Z^{-1} \sum_{m, n}\left|A_{m n}\right|^{2}\left(e^{-\beta E_{n}}-e^{-\beta E_{m}}\right) \beta\left(E_{m}-E_{n}\right)\right\}^{1 / 2} .
\end{aligned}
$$

The last factor can be recombined to give $\left(\beta\left\langle\left[A,\left[H, A^{+}\right]\right]\right\rangle\right)^{1 / 2}$. Finally:

$$
(A, A) \leqq \frac{1}{2}\left\langle\left[A, A^{+}\right]_{+}\right\rangle \leqq(A, A)+\frac{1}{2}\left\{\beta(A, A)\left\langle\left[A,\left[H, A^{+}\right]\right]\right\rangle\right\}^{1 / 2} .
$$

We now complete the proof of Lemma 4.

Suppose that $H$ depends linearly on some parameter $\alpha$. Let $H=H_{0}+\alpha A$ and suppose that $A$ is sufficiently well behaved for $p$ to have a second derivative with respect to $\alpha$. Then it can be shown [9] that:

$$
\frac{d^{2} p}{d \alpha^{2}}=\beta V^{-1}(A-\langle A\rangle, A-\langle A\rangle) \geqq 0 .
$$

Therefore $p$ is a convex function of $\alpha$. This applies in particular to $\mu$ and $r$ and proves the convexity properties stated in Lemma 4 . We now prove that $p$ is an increasing function of $r$. From now on we set $r=|\nu| \geqq 0$. Since $p$ does not depend on the argument $\omega$ of $\nu$, we choose $\omega=0$. Then

$$
[H, N]=-r V^{1 / 2}\left(a_{0}-a_{0}^{+}\right) \text {. }
$$

From $\langle[H, N]\rangle=0$, it follows that $\left\langle a_{0}\right\rangle=\left\langle a_{0}^{+}\right\rangle$(for $r=0$, both are zero, because of gauge invariance), so that $\left\langle a_{0}\right\rangle$ is real. Furthermore;

$$
[N,[H, N]]=r V^{1 / 2}\left(a_{0}+a_{0}^{+}\right) \text {. }
$$

From (2.5) with $A=N$ and from the reality of $\left\langle a_{0}\right\rangle$, we conclude that $\left\langle a_{0}\right\rangle \geqq 0$. Therefore:

$$
\frac{d p}{d r}=V^{-1 / 2}\left(\left\langle a_{0}\right\rangle+\left\langle a_{0}^{+}\right\rangle\right) \geqq 0 .
$$

Therefore $p$ is an increasing function of $r$, which completes the proof of Lemma 4.

\section{The B.A. for the Statistical Operator}

In this section, we consider the B.A. for the statistical operator $W$ (1.8). We first give a more precise definition of the B.A. Let $\psi_{0}$ be the one particle constant wave function: $\psi_{0}(x)=V^{-1 / 2} . \psi_{0}$ generates a one dimensional subspace of the one particle space. Let $\mathscr{H}_{0}$ be the symmetric 
tensor algebra constructed on this subspace, and $\mathscr{H}^{\prime}$ the symmetric tensor algebra constructed on the orthogonal complement of $\psi_{0}$ in the one particle space. Then the Fock space $\mathscr{H}$ is isomorphic to the tensor product:

$$
\mathscr{H} \approx \mathscr{H}_{0} \otimes \mathscr{H}^{\prime} .
$$

We consider the coherent states in $\mathscr{H}_{0}$, defined by [18]:

$$
|C\rangle=\exp \left(-|C|^{2} / 2\right) \sum_{l \geqq 0} \frac{C^{l}}{\sqrt{l !}}|l\rangle
$$

where $|l\rangle \in \mathscr{H}_{0}$ is the normalized $l$ particle state, and $C$ is any complex number. The phases of the states $|l\rangle$ are chosen in such a way that: $a_{0}|l\rangle=\sqrt{l}|l-1\rangle$. Then:

$$
a_{0}|C\rangle=C|C\rangle \text {. }
$$

We now define the B.A. as follows. To every operator $A$ on $\mathscr{H}$ and any complex number $C$, we associate the operator $A_{0}(C)$ on $\mathscr{H}^{\prime}$ defined by:

$$
A_{0}(C)=\langle C|A| C\rangle
$$

provided it exists. More precisely, if $\psi_{1}^{\prime}, \psi_{2}^{\prime} \in \mathscr{H}^{\prime}$, then the vectors $\psi_{1}^{\prime} \otimes|C\rangle \equiv\left|\psi_{1}^{\prime} \otimes C\right\rangle$ and $\psi_{2}^{\prime} \otimes|C\rangle \equiv\left|\psi_{2}^{\prime} \otimes C\right\rangle$ lie in $\mathscr{H}$, and:

$$
\left\langle\psi_{1}^{\prime}\left|A_{0}(C)\right| \psi_{2}^{\prime}\right\rangle=\left\langle\psi_{1}^{\prime} \otimes C|A| \psi_{2}^{\prime} \otimes C\right\rangle .
$$

Intuitively, the transformation $A \rightarrow A_{0}(C)$ consists in replacing $a_{0}$ and $a_{0}^{+}$by $C$ and $\bar{C}$ in $A$, after $A$ has been expanded as a normally ordered polynomial or power series in $a_{0}$ and $a_{0}^{+}$. (3.4) is therefore a reasonable definition of the B.A.

If $A$ is bounded, then $A_{0}(C)$ exists and is bounded. In fact:

$$
\| A_{0}(C)\left|\psi^{\prime}\right\rangle\|=\| A\left|C \otimes \psi^{\prime}\right\rangle\|\leqq\| A\|\| \psi^{\prime} \| \text {. }
$$

If $A$ is positive, $A_{0}(C)$ is positive. If $A$ is a positive trace class operator, then $A_{0}(C)$ has the same properties. In fact, $|C\rangle$ can be taken as the first vector of an orthonormal basis in $\mathscr{H}_{0}:\left(u_{1}=|C\rangle, \ldots, u_{n}, \ldots\right)$. Let $\left(v_{n}\right)$ be an orthonormal basis in $\mathscr{H}^{\prime}$. Then

$$
\operatorname{Tr}^{\prime} A_{0}(C)=\sum_{n}\left\langle u_{1} \otimes v_{n}|A| u_{1} \otimes v_{n}\right\rangle \leqq \operatorname{Tr} A
$$

where $\operatorname{Tr}^{\prime}$ means trace in $\mathscr{H}^{\prime}$.

On the other hand, if $A$ is unbounded, then $\left|C \otimes \psi^{\prime}\right\rangle$ may not be in the domain of $A$ for some or all $C$ complex and $\psi^{\prime} \in \mathscr{H}^{\prime}$, and $A_{0}(C)$ may not exist. This problem will arise in Section 5.

Let now $H$ and $W$ be defined by $(1.6,1.8)$. The B.A. transforms $W$ into a new operator $W_{0}(C)=\langle C|W| C\rangle$. We define:

$$
\begin{aligned}
Z_{0}(C) & =\operatorname{Tr}^{\prime} W_{0}(C) \\
\beta p_{0}(C) & =V^{-1} \log Z_{0}(C) .
\end{aligned}
$$

It follows immediately from (3.7) that $Z_{0}(C) \leqq Z$ and $p_{0}(C) \leqq p$. 
The unit operator $1_{0}$ in $\mathscr{H}_{0}$ has the integral representation [18]:

$$
\mathbf{1}_{\mathbf{0}}=\frac{\mathbf{1}}{2 \pi} \int d C d \bar{C}|C\rangle\langle C|
$$

where $d C d \bar{C}=2 d(\operatorname{Re} C) d(\operatorname{Im} C)$. Therefore, for any trace class operator $A$ on $\mathscr{H}_{0}$ :

$$
\operatorname{Tr}(A)=\frac{1}{2 \pi} \int d C d \bar{C}\langle C|A| C\rangle .
$$

Substituting (3.11) in the definition of $Z$, we obtain:

Therefore:

$$
Z=\operatorname{Tr} W=\operatorname{Tr}^{\prime} \frac{1}{2 \pi} \int d C d \bar{C}\langle C|W| C\rangle .
$$

$$
Z=\frac{1}{2 \pi} \int d C d \bar{C} Z_{0}(C) .
$$

(3.13) is the starting point both for proving the asymptotic exactness of the B.A. and for some qualitative remarks which we present now. We define a probability distribution in the complex plane $w(C) d C d \bar{C}$ by

$$
w(C)=(2 \pi Z)^{-1} Z_{0}(C) .
$$

Averages with respect to this distribution will be denoted by $\langle\langle\rangle$.

$$
\langle\langle(C)\rangle\rangle=\int w(C) f(C) d C d \bar{C} .
$$

For $v=0$, it follows from gauge invariance that $w$ depends only on $R=|C|$, and therefore $\langle\langle C\rangle\rangle=0$. This is no longer true for $v \neq 0$, but in any case:

$$
\left\langle a_{0}\right\rangle=Z^{-1} \operatorname{Tr}\left(a_{0} W\right)=Z^{-1} \frac{1}{2 \pi} \int d C d \bar{C} \operatorname{Tr}\left\langle C\left|W a_{0}\right| C\right\rangle .
$$

Therefore:

Similarly

$$
\left\langle a_{0}\right\rangle=\langle\langle C\rangle\rangle
$$

$$
\left\langle a_{0} a_{0}^{+}\right\rangle=Z^{-1} \frac{1}{2 \pi} \int d C d \bar{C} \operatorname{Tr}^{\prime}\left\langle C\left|a_{0}^{+} W a_{0}\right| C\right\rangle .
$$

Therefore the fluctuation of $C$ is closely related to that of $a_{0}$ :

$$
\left\langle\left(a_{0}^{+}-\left\langle a_{0}^{+}\right\rangle\right)\left(a_{0}-\left\langle a_{0}\right\rangle\right)\right\rangle=\left\langle\left\langle|C-\langle\langle C\rangle\rangle|^{2}\right\rangle\right\rangle-1 \text {. }
$$

The presence or absence of condensation is expected to reflect itself in the qualitative behavior of $w(C)$. If condensation does not occur, $w$ should be strongly peaked near the origin, both for $\nu=0$ and $\nu \neq 0$. $\left\langle\langle C\rangle\right.$ and $\left\langle\left\langle|C-\langle\langle C\rangle\rangle|^{2}\right\rangle\right\rangle$ should be small (i.e. of the order of 1 ). If condensation occurs, for $\nu=0, w$ should be concentrated in a neighborhood of a circle with radius of the order of $V^{1 / 2}$. One should then get, besides $\langle\langle C\rangle\rangle=0,\left\langle\left\langle\mid C-\left\langle\left.\langle C\rangle\right|^{2}\right\rangle\right\rangle\right.$ of the order of $V$. If $\nu \neq 0, w$ should be strongly peaked for $|C|$ of the order of $V^{1 / 2}$ and $\operatorname{Arg} C \approx \operatorname{Arg} \nu$. One should then have $\langle\langle C\rangle\rangle$ of the order of $V^{1 / 2}$, and $\left\langle\left\langle|C-\langle\langle C\rangle\rangle|^{2}\right\rangle\right\rangle$ small (i.e. of the order of 1$)$. 
We now return to the comparison of $p$ with $\operatorname{Sup} p_{0}(C)$. The problem is similar to the comparison of the grand canonical ensemble with the canonical one. In the latter case, we replace $\sum_{n}$ by Sup. In the former, we want to replace $(2 \pi)^{-1} \int d C d \bar{C}$ by $\operatorname{Sup}_{C}$. We now state the result. Theorem 2.

$$
\lim _{V \rightarrow \infty} \operatorname{Sup}_{C} p_{0}(C)=\lim _{V \rightarrow \infty} p(=\tilde{p})
$$

The proof is contained in Appendix A.

There is a great similarity between the B.A. and the $x$-ensemble description of many boson systems by LEE and YANG [19]. Let:

$$
W_{0}=\operatorname{Tr}^{\prime} W \text {. }
$$

Then $W_{0}$ is a positive trace class operator in $\mathscr{H}_{0}$. Moreover

$$
\left\|W_{0}\right\| \leqq \operatorname{Tr}_{0} W_{0}=Z
$$

where $\operatorname{Tr}_{0}$ means Trace in $\mathscr{H}_{0}$. Then LEE and YANG's proposal is to replace:

by :

$$
Z=\sum_{l}\left\langle l\left|W_{0}\right| l\right\rangle
$$

$$
Z_{x}=\exp (-x V) \sum_{l} \frac{(x V)^{l}}{l !}\left\langle l\left|W_{0}\right| l\right\rangle
$$

In the case where $H$ commutes with the particle number operator, i.e., for $v=0$, it is easily seen that $Z_{x}=Z_{0}(C)$ for $x=|C|^{2} / V$, so that LEE and YANG's proposal is identical with the B.A. (This is no longer true for $\nu \neq 0$ ). Theorem 2 then asserts that:

$$
\lim _{V \rightarrow \infty} \sup _{x} p_{x}=\lim _{V \rightarrow \infty} p=\tilde{p} \text {. }
$$

Notice however that this statement is weaker than those contained in ref. [19], Appendix A.

\section{The State of the System}

We make only a few simple or qualitative remarks. We consider first a finite system. The natural operator algebra associated with this system is the algebra $\mathfrak{A}$ of bounded operators in $\mathscr{H}$. Let $\mathfrak{A}^{\prime}$ be the subalgebra of bounded operators in $\mathscr{H}^{\prime}$. The state of the system is the positive normalized linear form $\varrho$ on $\mathfrak{A}$ defined by:

$$
\varrho(A)=Z^{-1} \operatorname{Tr}(A W)
$$

for any $A \in \mathfrak{A}$. Let $\varrho^{\prime}$ be the restriction of $\varrho$ to $\mathfrak{A}^{\prime}$. Then for any $A \in \mathfrak{A}^{\prime}$, we obtain, by use of (3.10):

$$
\varrho^{\prime}(A)=Z^{-1} \frac{1}{2 \pi} \int d C d \bar{C} \operatorname{Tr}^{\prime}\left(A W_{0}(C)\right) .
$$


We first take $v=0$. Let $C=R e^{i \theta}$. Then $Z_{0}(C)$ depends only on $R$, but not on $\theta$. Therefore:

Otherwise stated:

$$
\varrho^{\prime}(A)=\frac{1}{2 \pi} \int_{0}^{2 \pi} d \theta \frac{\int_{0}^{\infty} 2 R d R \operatorname{Tr}^{\prime}\left(A W_{0}\left(R e^{i \theta}\right)\right)}{\int_{0}^{\infty} 2 R d R \operatorname{Tr}^{\prime} W_{0}(R)} .
$$

$$
\varrho^{\prime}=\frac{1}{2 \pi} \int_{0}^{2 \pi} \varrho_{\theta}^{\prime}
$$

where $\varrho_{\theta}^{\prime}(A)$ is defined by the integrand in (4.3). This exhibits $\varrho^{\prime}$ as a convex combination (in fact an integral over the gauge group) of states $\varrho_{\theta}^{\prime}$ which are not gauge invariant, and can be obtained from one of them by gauge transformations. If $A$ is gauge invariant, then $\varrho^{\prime}(A)=\varrho_{\theta}^{\prime}(A)$ for any $\theta$. If $A$ belongs to an irreducible non-trivial representation of the gauge group, more precisely if:

$$
e^{i \alpha N} A e^{-i \alpha N}=e^{i \alpha n} A
$$

with $n \neq 0$, then $\varrho^{\prime}(A)=0$, whereas $\varrho_{\theta}^{\prime}(A)$ will not be zero in general.

In the infinite volume limit, one is faced with two problems.

(1) The problem of the exactness of the B.A. now consists in proving that the state $\varrho_{\theta}^{\prime}$ has the same limit as the state defined by the density matrix $Z_{0}(C)^{-1} W_{0}(C)$, where $C=R e^{i \theta}$ and $R$ is determined by the condition that $Z_{0}(C)=Z_{0}(R)$ be maximum.

(2) The more interesting question is to decide whether there is condensation and breakdown of the gauge invariance or not. In the former case, the common limit of $\varrho_{\theta}^{\prime}$ and of its Bogoliubov approximation will be non-gauge invariant and therefore will differ from the limit of $\varrho^{\prime}$ itself. In the latter case, both limits will coincide and therefore be gauge invariant. In both cases, (4.4) will remain true in the limit. Such a decomposition has been obtained in the case of the infinite free boson system by Arakr and Woods [20].

We now consider the case $\nu \neq 0$. This was the original proposal of Bogoliubov to obtain directly the non-gauge invariant states by a limiting process from $\varrho^{\prime}$ itself. This gives rise to a second equivalence problem, namely that of proving that the limit for $\nu \rightarrow 0$ (with $\nu=r e^{i \theta}$, $\theta$ fixed and $r \rightarrow 0$ ) of the infinite volume limit of the new $\varrho^{\prime}$ coincides with the infinite volume limit of the above $\varrho_{\theta}^{\prime}$. The preceding remarks show however that this procedure is not the only possible one to obtain the limiting non-gauge invariant states. It is moreover more natural to start with $\varrho_{\theta}^{\prime}(v=0)$, for which the decomposition (4.4) exists already for a finite system. 
We have restricted our attention to $\mathfrak{A}^{\prime}$. This restriction, however, should be unimportant, because the B.A. which is expected to be exact in the limit, consists precisely in eliminating $\mathscr{H}_{0}$, and therefore the distinction between $\mathfrak{A}$ and $\mathfrak{U}^{\prime}$.

\section{The B.A. in the Hamiltonian}

The original proposal of Bogoliubov $[1,5]$ was to replace $a_{0}$ and $a_{0}^{+}$by $C$-numbers in $H$ itself, and not in $W$. We therefore tentatively define:

$$
\begin{aligned}
H_{0}(C) & =\langle C|H| C\rangle, \\
W_{0}^{\prime}(C) & =\exp \left[-\beta H_{0}(C)\right], \\
Z_{0}^{\prime}(C) & =\operatorname{Tr} W_{0}^{\prime}(C), \\
\beta p_{0}^{\prime}(C) & =V^{-1} \log Z_{0}^{\prime}(C) .
\end{aligned}
$$

We are faced however with the difficulty that $H_{0}(C)$ may not exist. As a function of $a_{0}$ and $a_{0}^{+}, H$ is a polynomial of second degree in each of these operators. In particular it contains a term proportional to

$$
a_{0}^{+2} \int \phi(x-y) d x d y a_{0}^{2}
$$

which becomes infinite if $\phi$ is not integrable when $a_{0}$ and $a_{0}^{+}$are replaced by $c$-numbers. In this entire section we therefore impose on the potential $\phi$, besides (A), (B) and (C) of Section 1, the additional condition (D):

(D) $\phi$ is absolutely integrable:

$$
\int|\phi(x)| d x=\Phi<+\infty .
$$

Conditions (A) and (D) can be shown to be sufficient to define $H_{0}(C)$ as a self-adjoint operator in $\mathscr{H}^{\prime}$. As compared with (B) and (C), condition (D) is not substantially stronger as far as the behavior at large distances is concerned. It is however a restriction on the behavior of $\phi$ at the origin. For instance, it excludes hard cores. Intuitively, (D) implies that the Born approximation to the scattering of particles in the zero momentum one particle state is finite. By considering the operator $W_{0}^{\prime}(C)$ instead of $W_{0}(C)$, we are in fact considering the exponential of the Born approximation to this process, instead of the appropriate binary kernel [21], which is well defined for potentials satisfying (A), (B) and (C).

We next prove the following result:

Lemma 5.

$$
p_{0}^{\prime}(C) \leqq p_{0}(C) .
$$

Proof. It follows from Lemma $\mathrm{R} 2$ that:

$$
Z_{0}^{\prime}(C)=\operatorname{Tr}^{\prime} \exp \left[-\beta H_{0}(C)\right]=\operatorname{Sup}_{\left\{\psi^{\prime}\right\}} \sum_{\mathbf{i}} \exp \left[-\beta\left\langle\psi_{\mathrm{i}}^{\prime}\left|H_{0}(C)\right| \psi_{\mathrm{i}}^{\prime}\right\rangle\right]
$$

where the Sup is taken over all possible orthonormal families of vectors 
in the domain of $H_{0}(C)$. Therefore:

$$
Z_{0}^{\prime}(C)=\operatorname{Sup}_{\left\{\psi^{\prime}\right\}} \sum_{\mathrm{i}} \exp \left\{-\beta\left\langle C \otimes \psi_{\mathrm{i}}^{\prime}|H| C \otimes \psi_{\mathrm{i}}^{\prime}\right\rangle\right\} .
$$

Now for any self-adjoint operator $A$ bounded from below and with discrete spectrum, we have [22]:

Therefore:

$$
\exp (-\langle\psi|A| \psi\rangle) \leqq\langle\psi|\exp (-A)| \psi\rangle .
$$

$$
Z_{0}^{\prime}(C) \leqq \operatorname{Sup}_{\left\{\psi^{\prime}\right\}} \sum_{\mathbf{i}}\left\langle C \otimes \psi_{\mathbf{i}}^{\prime}|\exp (-\beta H)| C \otimes \psi_{\mathrm{i}}^{\prime}\right\rangle=Z_{0}(C) .
$$

The lemma follows immediately.

We now obtain further bounds for $p_{0}^{\prime}(C)$. Using again (5.10), we obtain, for any $\psi^{\prime}$ in the domain of $H_{0}(C)$ :

$$
Z_{0}^{\prime}(C) \geqq \exp \left[-\beta\left\langle C \otimes \psi^{\prime}|H| C \otimes \psi^{\prime}\right\rangle\right] .
$$

We take $\psi^{\prime}$ to be the zero particle state in $\mathscr{H}^{\prime}$. Then:

$$
p_{0}^{\prime}(C) \geqq \mu \frac{|C|^{2}}{V}-\Phi \frac{|C|^{4}}{V^{2}}+\frac{\bar{\nu} C+\nu \bar{C}}{\sqrt{\bar{V}}} .
$$

In the $(\mu, p)$ plane, this is a straight line with positive slope. For $v=0$, it remains tangent to a fixed parabola as $C$ varies (see Fig. 2).

An upper bound for $p_{0}^{\prime}(C)$ can be easily deduced from the stability condition (B). By the same method as in Section 1, one obtains:

$$
p_{0}^{\prime}(C) \leqq(\mu+B) \frac{|C|^{2}}{V}-A \frac{|C|^{4}}{V^{2}}+\frac{\bar{\nu} C+\nu \bar{C}}{\sqrt{\bar{V}}}+\hat{p}(\mu, 0) .
$$

From the behavior of this bound as $\mu \rightarrow-\infty$ and from the convexity of $p_{0}^{\prime}(C)$, one easily constructs an upper bound for $p_{0}^{\prime}(C)$, represented in the $(\mu, p)$ plane by the left part of the tangent with slope $|C|^{2} / V$ to the

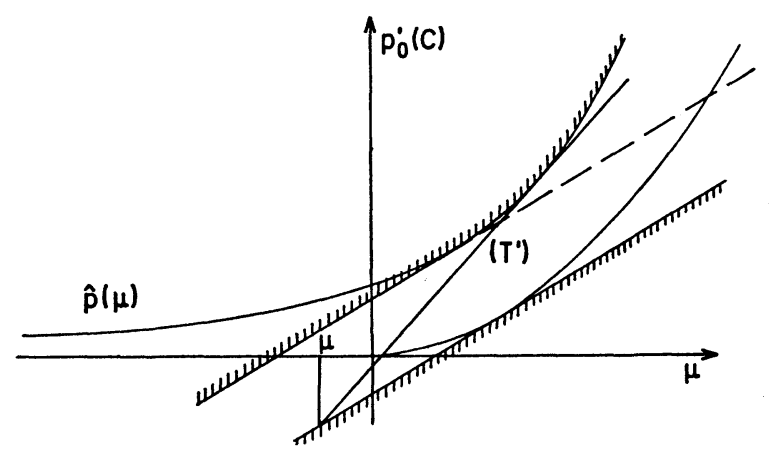

Fig. 2. Upper and lower bounds for $p_{0}^{\prime}(C)$ for fixed $C . \hat{\varrho}_{0}\left(\frac{|C|^{2}}{V}, \mu\right)=$ slope of $\left(T^{\prime}\right)$ 
curve of $\hat{p}(\mu)$ and the right part of the latter, left and right being defined with respect to the contact point (Fig. 2). From the convexity of $p_{0}^{\prime}(C)$ and the lower bound (5.13), one obtains by the same construction as in Section 1 an upper bound $\hat{\varrho}_{0}\left(\frac{|C|^{2}}{V}, \mu\right)$ for $\varrho_{0}^{\prime}(C)=\partial p_{0}^{\prime}(C) / \partial \mu$. As a function of $\mu, \hat{\varrho}_{0}$ tends to $\frac{|C|^{2}}{V}$ as $\mu \rightarrow-\infty$, and is smaller than $\hat{\varrho}(\mu)$ for $\mu \rightarrow+\infty$.

It follows from Lemma 5 that $p_{0}^{\prime}(C)$ is a worse approximation to $p$ than $p_{0}(C)$. This was already expected from the need of the supplementary condition (D) and its intuitive interpretation.

As previously, $C$ should be chosen such that $p_{0}^{\prime}(C)$ be maximum. If $\nu=0, p_{0}^{\prime}(C)$ depends only on $R=|C|$ because of gauge invariance, and the maximum condition at most determines $R$. If $\nu \neq 0$, it follows from the relation:

$$
p_{0}^{\prime}(C, \nu)=p_{0}^{\prime}(C, 0)+V^{-1 / 2}(\bar{C} \nu+C \bar{\nu})
$$

that the maximum condition determines the argument of $C$ as being equal to that of $\nu$. If we assume that $p_{0}^{\prime}(C)$ is a differentiable function of $C$, we obtain as a necessary condition for $p$ to be maximum:

$$
\left\langle\frac{\partial H_{0}(C)}{\partial C}\right\rangle_{H_{0}(C)} \equiv\left\langle\frac{\partial U_{0}(C)}{\partial C}\right\rangle_{H_{0}(C)}-\mu \bar{C}-\bar{\nu} V^{1 / 2}=0
$$

where $U_{0}(C)=\langle C|U| C\rangle$ and the average is taken with the density matrix $Z_{0}^{\prime}(C)^{-1} W_{0}^{\prime}(C)$. This equation, already considered by BogolIUBov [5] is the analog in the present problem of the gap equation of the BCS model. If $v=0$, then $H_{0}(0)$ is gauge invariant whereas in $U_{0}(C)$ the term linear in $C$ is not. Therefore $\left\langle\left.\frac{\partial U_{0}}{\partial C}\right|_{C=0}\right\rangle=0$ so that (5.16) for $v=0$ always has the trivial solution $C=0$. This is also in strong analogy with the BCS gap equation. Notice finally that for free bosons, (5.16) takes the degenerate form $\mu C=0$. Condensation can occur only at $\mu=0$, and the density $|C|^{2} / V$ of the condensate is not determined by the equation. This again illustrates the pathology associated with free bosons.

We now compare $p_{0}^{\prime}(C)$ to $p$ in the infinite volume limit. Theorem 3.

$$
\lim _{V \rightarrow \infty} \operatorname{Sup}_{C} p_{0}^{\prime}(C)=\lim _{V \rightarrow \infty} p \equiv \tilde{p}
$$

The remaining part of Section 5 is devoted to the proof of Theorem 3. This proof is a transposition to the boson case of that of the corresponding property for the BCS model, given in ref. [9]. It involves two steps. We consider the function of $r=|\nu|$ :

$$
\Delta(r)=p-\operatorname{Sup}_{C} p_{0}^{\prime}(C) .
$$


In a first step, we obtain a bound for this function in terms of the fluctuation of $a_{0}$. In a second step, we relate this fluctuation to the second derivative of $p$ with respect to $r$, obtain bounds both for the derivative of $\Delta$ with respect to $r$ and its integral over $r$, and deduce therefrom bounds for $\Delta$ itself. A number of formulas needed for the proof are listed in Appendix B. In what follows, the notation $\langle A\rangle_{B}$ means $\operatorname{Tr}\left\langle A e^{-\beta B}\right) / \operatorname{Tr}\left(e^{-\beta B}\right)$. If the subscript $B$ does not appear, it is understood that $B$ is $H$ itself.

Let $h_{0}$ be a positive operator in $\mathscr{H}_{0}$, to be chosen later. In order to compare $p_{0}^{\prime}(C)$ with $p$, we introduce the interpolating function $q(t)$ :

$$
\beta q(t)=V^{-1} \log \operatorname{Tr} \exp (-\beta H(t))
$$

where $H(t)$ is the following operator in $\mathscr{H}$ :

Then

$$
H(t)=(1-t)\left(H_{0}(C)+h_{0}\right)+t H .
$$

$$
\left\{\begin{array}{l}
q(1)=p \\
q(0)=p_{0}^{\prime}(C)+(\beta V)^{-1} \log \left[\operatorname{Tr} \exp \left(-\beta h_{0}\right)\right] .
\end{array}\right.
$$

It follows from Lemma 3 in Section 1 that for any fixed $\Lambda, q(t)$ is an analytic function of $t$ for $0<\operatorname{Re} t<1$. For real $t$, $(0<t<1)$ its derivative is given by:

$$
\frac{d q}{d t}=V^{-1}\left\langle H_{0}(C)+h_{0}-H\right\rangle_{H(t)} .
$$

This quantity has well defined limits as $t \rightarrow 0^{+}$and $t \rightarrow 1^{-}$. We shall now choose $h_{0}$ such that $d q / d t \geqq 0$ for $t=0$. Then $q(t)$ will be an increasing function of $t$. Therefore:

and:

$$
p_{0}^{\prime}(C) \leqq q(0) \leqq q(1)=p
$$

$$
q(1)-q(0) \leqq d q|d t|_{t=1} \text {. }
$$

This provides a second proof of the inequality $p_{0}^{\prime}(C) \leqq p$ and an upper bound to the major part of the difference $p-p_{0}^{\prime}(C)$. The best bound will be obtained for the smallest $h_{0}$ for which $d q / d t \geqq 0$ at $t=0$. We look for $h_{0}$ in the form:

$$
h_{0}=K\left(a_{0}^{+}-\bar{C}\right)\left(a_{0}-C\right)+L\left(a_{0}^{+}-\bar{C}\right)^{2}\left(a_{0}-C\right)^{2}
$$

with $K$ and $L$ positive constants. It follows from (5.25) that for $n \neq p$ :

$$
\left\langle\left(a_{0}^{+}-\bar{C}\right)^{n}\left(a_{0}-C\right)^{p}\right\rangle_{h_{0}}=0 .
$$

From a Taylor expansion of $H$ near $H_{0}(C)$, we then obtain:

$$
\begin{aligned}
\left\langle H_{0}(C)+h_{0}-H\right. & \rangle_{H_{0}(C)+h_{0}}=K-\left\langle\frac{\partial^{2} H_{0}(C)}{\partial C \partial \bar{C}}\right\rangle_{H_{0}(C)}\left\langle\left(a_{0}^{+}-\bar{C}\right)\left(a_{0}-C\right)\right\rangle_{h_{0}} \\
+ & \left(L-\frac{1}{4} \frac{\partial^{4} H_{0}(C)}{\partial^{2} C \partial^{2} \bar{C}}\right)\left\langle\left(a_{0}^{+}-\bar{C}\right)^{2}\left(a_{0}-C\right)^{2}\right\rangle_{h_{0}} .
\end{aligned}
$$


Using (B.5), we make the last term vanish by choosing:

$$
L=\frac{1}{4} \frac{\partial^{4} H_{0}(C)}{\partial^{2} C \partial^{2} \bar{C}}=\left(2 V^{2}\right)^{-1} \int_{\Lambda} d x d y \phi(x-y) .
$$

$L$ is bounded by $\Phi / 2 V$, because of condition (D). From (B.2), we obtain:

$$
\frac{\partial^{2} H_{0}(C)}{\partial C \partial \bar{C}}=\left\langle C\left|\left[a_{0}\left[H, a_{0}^{+}\right]\right]\right| C\right\rangle \text {. }
$$

Now, it follows from (B.3) that:

Therefore:

$$
\left[a_{0},\left[H, a_{0}^{+}\right]\right] \leqq 2 \Phi V^{-1} N-\mu \text {. }
$$

$$
\left\langle\frac{\partial^{2} H_{0}}{\partial C \partial \bar{C}}\right\rangle_{H_{0}(C)} \leqq 2 \Phi \hat{\varrho}_{0}\left(\frac{|C|^{2}}{V}, \mu\right)-\mu \text {. }
$$

On the other hand, we obtain from (2.5) and (5.30):

We then choose:

$$
0 \leqq\left\langle\left[a_{0},\left[H, a_{0}^{+}\right]\right]\right\rangle \leqq 2 \Phi \hat{\varrho}(\mu)-\mu \text {. }
$$

$$
K=-\mu+2 \Phi \max \left(\hat{\varrho}(\mu), \hat{\varrho}_{0}\left(\frac{|C|^{2}}{V}, \mu\right)\right)+1 .
$$

It follows from $(5.27,28,31,33)$ that $d q / d t \geqq 0$ at $t=0$. Furthermore, from (5.32), we know that $K \geqq 1$. Therefore, the second term in the RHS of (5.21) is smaller than:

$$
\begin{aligned}
(\beta V)^{-1} \log \operatorname{Tr}_{0} \exp \left[-\beta\left(a_{0}^{+}-\bar{C}\right)\left(a_{0}-C\right)\right] & \\
& =-(\beta V)^{-1} \log \left(1-e^{-\beta}\right) .
\end{aligned}
$$

Therefore $q(0)-p_{0}^{\prime}(C)$ tends to zero as $V \rightarrow \infty$.

The next step in the proof is to obtain an upper bound for $d q / d t$ at $t=1$ in terms of the fluctuation of $a_{0}$. We therefore consider $H_{0}(C)-H$, as given by (B.2). After some elementary manipulations, the terms of first and second order can be combined to give:

$$
\begin{aligned}
& -\frac{1}{2}\left[A^{+} A,\left[H, A^{+} A\right]\right]+2 A^{+}\left[A,\left[H, A^{+}\right]\right] A-\frac{3}{2} A^{+}[A, H] \\
& -\frac{3}{2}\left[H, A^{+}\right] A
\end{aligned}
$$

where

$$
A=a_{0}-C \text {. }
$$

Because of (2.5), the average of the first term is negative and can be dropped, since we are looking for an upper bound. Because of (5.30), the second term, which is hermitian, is bounded by:

$$
-2 \mu\left(a_{0}^{+}-\bar{C}\right)\left(a_{0}-C\right)+4 \Phi V^{-1}\left(a_{0}^{+}-\bar{C}\right) N\left(a_{0}-C\right) \text {. }
$$

We consider the last two terms. Their averages do not depend on $C$, and are real and equal. We have:

$$
-2\left\langle A^{+}[A, H]\right\rangle=\left\langle\left[A^{+},[H, A]\right]\right\rangle+\left\langle\left[A^{+},[H, A]\right]_{+}\right\rangle \text {. }
$$


The first term in the RHS is bounded by $2 \Phi \hat{\varrho}(\mu)-\mu$. Using a spectral decomposition of $H$, we write the second term as:

$$
\left\langle\left[A^{+},[H, A]\right]_{+}\right\rangle=\sum_{m, n}\left|A_{m n}\right|^{2}\left(E_{m}-E_{n}\right)\left(W_{n}+W_{m}\right)
$$

where $W_{n}=\exp \left(-\beta E_{n}\right)$. From (2.7), we then obtain:

$$
\begin{aligned}
\left|\left\langle\left[A^{+},[H, A]\right]_{+}\right\rangle\right| \leqq & \sum\left|A_{m n}\right|^{2}\left|E_{m}-E_{n}\right|\left|W_{n}-W_{m}\right| \\
& +2 \beta^{-1} \sum\left|A_{m n}\right|^{2}\left|W_{n}-W_{m}\right| \\
\leqq & \left\langle\left[A,\left[H, A^{+}\right]\right]\right\rangle+2 \beta^{-1}\left\langle\left[A, A^{+}\right]_{+}\right\rangle
\end{aligned}
$$

The third and fourth order terms in $H_{0}(C)+h_{0}-H$ are bounded by:

$$
\Phi V^{-1}|C|^{2}\left\langle\left(a_{0}^{+}-\bar{C}\right)\left(a_{0}-C\right)\right\rangle \text {. }
$$

Collecting $(5.25,37,38,41,42)$ we obtain finally:

$$
\begin{aligned}
\left\langle H_{0}(C)\right. & \left.+h_{0}-H\right\rangle \leqq 3(2 \Phi \hat{\varrho}(\mu)-\mu)+3 \beta^{-1}\left\langle\left[\left(a_{0}^{+}-\bar{C}\right),\left(a_{0}-C\right)\right]_{+}\right\rangle \\
& +(K-2 \mu)\left\langle\left(a_{0}^{+}-\bar{C}\right)\left(a_{0}-C\right)\right\rangle+4 \Phi V^{-1}\left\langle\left(a_{0}^{+}-\bar{C}\right) N\left(a_{0}-C^{\prime}\right)\right\rangle \\
& +\Phi V^{-1}|C|^{2}\left\langle\left(a_{0}^{+}-\bar{C}\right)\left(a_{0}-C\right)\right\rangle .
\end{aligned}
$$

Now for given $\mu$, the maximum of $p_{0}^{\prime}(C)$ will certainly not occur for values of $C$ for which $p_{0}^{\prime}(C)<0$. It follows from (5.14) that there exists $\xi$ such that $p_{0}^{\prime}(C)<0$ for $|C|^{2}>\xi V$. We therefore restrict our attention to $|C|^{2} \leqq \xi V$. Then in (5.43), $K$ and the coefficient $V^{-1}|C|^{2}$ of the last term in the RHS are bounded uniformly with respect to $V$. On the other hand, it follows easily from the stability condition (B) and the bounds in Section 1 and Appendix A that there exist positive constants $u$ and $v$, independent of $V$, such that:

$$
V^{-1}\left\langle\left(a_{0}^{+}-\bar{C}\right) N\left(a_{0}-C\right)\right\rangle \leqq u+v\left\langle\left(a_{0}^{+}-\bar{C}\right)\left(a_{0}-C\right)\right\rangle .
$$

Therefore, it follows from (5.43) and (5.21) that for all $C$ such that $|C|^{2}<\xi V$, there exist two positive constants $\gamma$ and $\delta$, independent of $V$, such that

$$
\begin{aligned}
p-p_{0}^{\prime}(C) & \leqq V^{-1}\left(\left\langle H_{0}(C)+h_{0}-H\right\rangle-\beta^{-1} \log \left(1-e^{-\beta}\right)\right) \\
& \leqq V^{-1}\left(\gamma+\frac{\delta}{2}\left\langle\left[\left(a_{0}^{+}-\bar{C}\right),\left(a_{0}-C\right)\right]_{+}\right\rangle\right) .
\end{aligned}
$$

The end of the proof of Theorem 3 is now very similar to that in ref. [9]. The best bound in (5.45) is obtained for $C=\left\langle a_{0}\right\rangle$. Therefore

$$
\begin{aligned}
0 & \leqq \Delta(r) \equiv p-\operatorname{Sup}_{C} p_{0}^{\prime}(C) \\
& \leqq V^{-1}\left(\gamma+\frac{\delta}{2}\left\langle\left[\left(a_{0}^{+}-\left\langle a_{0}^{+}\right\rangle\right),\left(a_{0}-\left\langle a_{0}\right\rangle\right)\right]_{+}\right\rangle\right) .
\end{aligned}
$$

Using (2.10) and (5.32), we obtain:

$$
\begin{aligned}
\Delta(r) & \leqq V^{-1}\left\{\gamma+\delta\left(a_{0}-\left\langle a_{0}\right\rangle, a_{0}-\left\langle a_{0}\right\rangle\right)+\frac{\delta}{2} \beta^{1 / 2}\right. \\
& \left.\cdot(2 \Phi \hat{\varrho}(\mu)-\mu)^{1 / 2}\left(a_{0}-\left\langle a_{0}\right\rangle, a_{0}-\left\langle a_{0}\right\rangle\right)^{1 / 2}\right\} .
\end{aligned}
$$


We take $\nu=\bar{v}=r$ to be real positive. Now:

$$
\left(a_{0}-\left\langle a_{0}\right\rangle, a_{0}-\left\langle a_{0}\right\rangle\right)=\beta^{-1} \frac{\partial^{2} p}{\partial \nu \partial \bar{\nu}}=(4 \beta r)^{-1} \frac{d}{d r}\left(r \frac{d p}{d r}\right) .
$$

Multiplying (5.47) by $r$ and integrating from $r_{1}$ to $r_{2}$, we obtain:

$$
\begin{aligned}
\int_{r_{1}}^{r_{3}} r \Delta(r) d r \leqq & V^{-1}\left\{\gamma \frac{r_{2}^{2}-r_{1}^{2}}{2}+\frac{\delta}{4 \beta}\left[r \frac{d p}{d r}\right]_{1}^{2}+\frac{\delta}{4}\right. \\
& \left.\cdot(2 \Phi \hat{\varrho}(\mu)-\mu)^{1 / 2} \int_{r_{1}}^{r_{3}}\left(r \frac{d}{d r} r \frac{d p}{d r}\right)^{1 / 2} d r\right\} .
\end{aligned}
$$

Schwarz' inequality gives for the last integral the bound:

Now:

$$
\left(\frac{r_{2}^{2}-r_{1}^{2}}{2}\right)^{1 / 2}\left(\left[r \frac{d p}{d r}\right]_{1}^{2}\right)^{1 / 2}
$$

Therefore:

$$
\frac{d p}{d r}=\frac{2\left\langle a_{0}\right\rangle}{V^{1 / 2}} \leqq 2 \hat{\varrho}(\mu, r)^{1 / 2}\left(\equiv 2 \hat{\varrho}^{1 / 2}\right) .
$$

$$
\begin{aligned}
\int_{r_{1}}^{r_{2}} r \Delta(r) d r & \leqq V^{-1}\left\{\gamma \frac{r_{2}^{2}-r_{1}^{2}}{2}+\frac{\delta}{2 \beta}\left(r_{1}+r_{2}\right) \hat{\varrho}^{1 / 2}\right. \\
& \left.+\frac{\delta}{4}(2 \Phi \hat{\varrho}-\mu)^{1 / 2} \hat{\varrho}^{1 / 4}\left(r_{2}^{2}-r_{1}^{2}\right)^{1 / 2}\left(r_{1}+r_{2}\right)^{1 / 2}\right\} .
\end{aligned}
$$

From (5.51) and the fact that the maximum of $p_{0}^{\prime}(C)$ occurs for $|C|^{2} \leqq \xi V$, we deduce:

Therefore, for $r>r_{1}$ :

$$
\left|\frac{d \Delta}{d r}\right| \leqq 2\left(\hat{\varrho}^{1 / 2}+\xi^{1 / 2}\right) .
$$

$$
\Delta\left(r_{1}\right) \leqq \Delta(r)+\left(r-r_{1}\right) 2\left(\hat{\varrho}^{1 / 2}+\xi^{1 / 2}\right) .
$$

We multiply both sides by $r$ and integrate over $r$ :

$$
\Delta\left(r_{1}\right) \frac{r_{2}^{2}-r_{1}^{2}}{2} \leqq \int_{r_{1}}^{r_{3}} r \Delta(r) d r+2\left(\hat{\varrho}^{1 / 2}+\xi^{1 / 2}\right) \frac{r_{1}+2 r_{2}}{6}\left(r_{2}-r_{1}\right)^{2} .
$$

We now compare (5.52) with (5.55) and replace $r_{1}$ by $r$ and $r_{2}$ by $r+\varepsilon$. Then:

$$
\begin{aligned}
\Delta(r) \leqq\left(\hat{\varrho}^{1 / 2}+\xi^{1 / 2}\right) \varepsilon \frac{r+2 \varepsilon / 3}{r+\varepsilon / 2} & +V^{-1}\left\{\gamma+\delta \beta^{-1} \hat{\varrho}^{1 / 2} \varepsilon^{-1}\right. \\
& \left.+\frac{\delta}{2}(2 \Phi \hat{\varrho}-\mu)^{1 / 2} \hat{\varrho}^{1 / 4} \varepsilon^{-1 / 2}\right\} .
\end{aligned}
$$

The best choice of $\varepsilon$ is $\varepsilon \approx V^{-1 / 2}$, which gives

$$
\Delta(r) \leqq \eta V^{-1 / 2}
$$

where $\eta$ is independent of $V$ and can be taken independent of $r$ in any bounded interval. Therefore $\Delta(r) \rightarrow 0$ as $V \rightarrow \infty$, and Theorem 3 is proved. 


\section{Conclusion}

We have proved the following results.

(1) For any potential $\phi$ satisfying (A), (B) and (C), $p_{0}(C)$ as defined by (3.9) satisfies:

$$
\begin{gathered}
p_{0}(C) \leqq p, \\
\lim _{V \rightarrow \infty}\left(p-\operatorname{Sup}_{C} p_{0}(C)\right)=0 .
\end{gathered}
$$

(2) For any potential satisfying (A), (B), (C) and (D), $p_{0}^{\prime}(C)$ as defined by (5.4) satisfies:

$$
\begin{gathered}
p_{0}^{\prime}(C) \leqq p_{0}(C) \leqq p, \\
\lim _{V \rightarrow \infty}\left(p-\operatorname{Sup}_{C} p_{0}^{\prime}(C)\right)=0 .
\end{gathered}
$$

Therefore the B.A. in either of the two forms which we have considered, gives the correct pressure in the infinite volume limit.

Clearly the results of Section 3 can be extended to the case where many body forces are present, provided they satisfy the appropriate generalizations of conditions (A), (B) and (C). On the other hand, the proof of Theorem 2 [i.e. of (6.4)], relies heavily on the pairwise additivity of the forces. It is very likely that many body forces would not impair the result, but the proof given here would rapidly become intractable.

We did not touch on the more interesting questions that arise for the state of the system, as sketched in the Introduction and in Section 4. This is certainly a much more difficult problem.

Acknowledgments. The author is grateful to Professor K. SyManzik and Professor W. ZIMMERmanN for the hospitality extended to him at the Courant Institute of Mathematical Sciences, where part of this work was done.

\section{Appendix A}

We prove Theorem 1. The proof consists in getting an upper bound for $Z$ in terms of $\max _{C} Z_{0}(C)$ by showing that the main contribution to the integral in (3.13) comes from a region of size $|C| \lesssim V^{1 / 2}$. We therefore show first that $Z_{0}(C)$ is small for large $R=|C|$. From (3.2), we have

$$
Z_{0}(C)=\sum_{l, l^{\prime}} e^{-|C|^{2}} \frac{C^{l}}{(l !)^{1 / 2}} \frac{\bar{C}^{\prime}}{\left(l^{\prime} !\right)^{1 / 2}}\left\langle l^{\prime}\left|W_{0}\right| l\right\rangle .
$$

Let $l_{0}=\alpha R^{2}$, where $0<\alpha<1$. We split the sums over $l, l^{\prime}$ according to the position of $l$ and $l^{\prime}$ with respect to $l_{0}$.

$$
Z_{0}(C) \leqq \sum_{l \leqq l_{0}}+\sum_{l^{\prime}} \sum_{\substack{l \\ l}}+\sum_{\substack{l>l_{0} \\ l^{\prime}>l_{0}}}
$$

where the positive sum over $l \leqq l_{0}, l^{\prime} \leqq l_{0}$ is counted twice. The sum of 
the first two terms recombines to:

$$
\begin{gathered}
2 \operatorname{Re} \sum_{l \leqq l_{0}} e^{-|C|^{2 / 2}} \frac{C^{l}}{(l !)^{1 / 2}}\left\langle C\left|W_{0}\right| l\right\rangle \\
\leqq 2\left\{\sum_{l \leqq l_{0}} e^{-|C|^{2}} \frac{|C|^{2 l}}{l !}\right\}^{1 / 2}\left\{\sum_{l \leqq l_{0}}\left|\left\langle C\left|W_{0}\right| l\right\rangle\right|^{2}\right\}^{1 / 2}
\end{gathered}
$$

where we have used Schwarz' inequality. Now

$$
\sum_{l \leqq l_{0}}\left|\left\langle C\left|W_{0}\right| l\right\rangle\right|^{2} \leqq\left\langle C\left|W_{0}^{2}\right| C\right\rangle \leqq\left\|W_{0}\right\|^{2} \leqq Z^{2} .
$$

Combining $(\mathrm{A} .3,4)$, we obtain for the first two terms in the RHS of (A.2) the bound

$$
2 Z \alpha^{1 / 2} R \exp \left[-R^{2}(1-\alpha+\alpha \log \alpha) / 2\right] .
$$

We now turn to the last term in (A.2). From Schwarz' inequality:

$$
\sum_{l, l^{\prime}>l_{0}} \cdots \leqq\left\{\sum_{l, l^{\prime}} \frac{R^{2 l}}{l !} \frac{R^{2 l^{\prime}}}{l^{\prime} !} e^{-2 R^{2}}\right\}^{1 / 2}\left\{\sum_{\substack{l>l_{0} \\ l^{\prime}>l_{0}}}\left|\left\langle l^{\prime}\left|W_{0}\right| l\right\rangle\right|^{2}\right\}^{1 / 2} .
$$

The RHS is bounded by:

$$
\left\{\sum_{l, l^{\prime}>l_{0}}\left\langle l\left|W_{0}\right| l\right\rangle\left\langle l^{\prime}\left|W_{0}\right| l^{\prime}\right\rangle\right\}^{1 / 2}=\sum_{l>l_{0}}\left\langle l\left|W_{0}\right| l\right\rangle .
$$

Now a vector in $\mathscr{H}$ representing a state where at least $l_{0}$ particles are in the one particle state $\psi_{0}$, is certainly orthogonal to any vector in $\mathscr{H}$ representing a state with less than $l_{0}$ particles. Therefore, if $P_{n}$ is the projection operator on the subspace of $\mathscr{H}$ defined by $N>n$, then:

$$
\sum_{l>l_{0}}\left\langle l\left|W_{0}\right| l\right\rangle \leqq \operatorname{Tr}\left(P_{l_{0}} W\right)
$$

For $v=0$, it follows from (1.34) that:

$$
\operatorname{Tr}\left(P_{l_{0}} W\right) \leqq \sum_{n>l_{0}} \exp \left\{V \lambda^{-\nu_{0}}+n[1+\beta(\mu+B)]-A \beta n^{2} V^{-1}\right\} .
$$

For $\nu \neq 0$, there is no such simple bound available. One can nevertheless prove by a more complicated argument that there exist constants $M, \eta$ and $\delta>0$, such that for $l_{0} \geqq \eta V$, one has

$$
\operatorname{Tr} P_{l_{0}} W \leqq M \exp \left(-\delta l_{0}\right) \text {. }
$$

$Z_{0}(C)$ is then bounded by the sum of (A.5) and (A.10).

We now turn to the proof of the theorem. We obtain from (3.13)

$$
Z \leqq R_{0}^{2} \max _{C} Z_{0}(C)+\frac{1}{2 \pi} \int_{|C|>R_{0}} d C d \bar{C} Z_{0}(C)
$$

where $R_{0}$ is a positive number. The last term in the RHS of (A.11) is bounded by the sum of two terms, which are the contributions from (A.5) and (A.10) to an upper bound for $Z_{0}(C)$. The first term is the 4 Commun. math. Phys., Vol. 8 
contribution from (A.5):

where

$$
\begin{aligned}
& 2 Z \alpha^{1 / 2} \int_{R_{0}}^{\infty} 2 R^{2} d R \exp \left(-\varepsilon R^{2}\right) \\
& \leqq 2 Z \alpha^{1 / 2} \frac{\varepsilon R_{0}^{2}+1}{\varepsilon^{2} R_{0}} \exp \left(-\varepsilon R_{0}^{2}\right)
\end{aligned}
$$

$$
\varepsilon=(1-\alpha+\alpha \log \alpha) / 2>0 .
$$

The second term is the contribution from (A.10). After an elementary calculation, we obtain the following upper bound for this term:

$$
M(\delta \alpha)^{-1} \exp \left[-\delta \alpha R_{0}^{2}\right]
$$

where we have chosen $\alpha R_{0}^{2}=\eta V$.

Comparing (A.11, 12, 14), we obtain:

$$
\begin{aligned}
Z(1 & \left.-2 \alpha^{1 / 2} \frac{\varepsilon R_{0}^{2}+1}{\varepsilon^{2} R_{0}} \exp \left(-\varepsilon R_{0}^{2}\right)\right) \\
& \leqq R_{0}^{2} \max _{C} Z_{0}(C)+M(\delta \alpha)^{-1} \exp \left(-\delta \alpha R_{0}^{2}\right)
\end{aligned}
$$

As $V \rightarrow \infty$, the coefficient of $Z$ in the LHS of (A.15) tends to one, and the remainder in the RHS is bounded uniformly with respect to $V$.

Theorem 2 follows immediately.

\section{Appendix B}

We list here some formulas which are needed for the proof of Theorem 3. The hamiltonian $H$ of the system is a normally ordered polynomial in $a_{0}$ and $a_{0}^{+}$, of second degree with respect to both variables. We can express $H_{0}(C)$ by a Taylor expansion around $a_{0}$. The derivatives of $H$ with respect to $a_{0}$ and $a_{0}^{+}$are obtained most easily by commutators with $a_{0}^{+}$and $a_{0}$. The two operations commute, because of Jacobi's identity and the relation $\left[a_{0}, a_{0}^{+}\right]=1$. Then

$$
\begin{aligned}
H=T & -\mu N-\sqrt{V}\left(\bar{\nu} a_{0}+v a_{0}^{+}\right) \\
& +\frac{1}{2} \int \phi(x-y) a^{+}(x) a^{+}(y) a(x) a(y) d x d y \\
H_{0}(C)=H & +\left(\bar{C}-a_{0}^{+}\right)\left[a_{0}, H\right]+\text { h.c. }+\frac{1}{2}\left(\bar{C}-a_{0}^{+}\right)^{2}\left[a_{0},\left[a_{0}, H\right]\right] \\
& + \text { h.c. }+\left(\bar{C}-a_{0}^{+}\right)\left[a_{0}\left[H, a_{0}^{+}\right]\right]\left(C-a_{0}\right) \\
& +\frac{1}{2}\left(\bar{C}-a_{0}^{+}\right)^{2}\left[a_{0},\left[a_{0},\left[H a_{0}^{+}\right]\right]\right]\left(C-a_{0}\right)+\text { h.c. } \\
& +\frac{1}{4}\left(\bar{C}-a_{0}^{+}\right)^{2}\left[a_{0},\left[a_{0},\left[\left[H, a_{0}^{+}\right] a_{0}^{+}\right]\right]\right]\left(C-a_{0}\right)^{2} .
\end{aligned}
$$


By order of a term in this expansion, we mean the total order of derivation (i.e. of commutation). We need only the explicit expressions of the higher order commutators, as listed below.

$$
\begin{gathered}
{\left[a_{0}\left[H, a_{0}^{+}\right]\right]=-\mu+V^{-1} \int_{A} \phi(x-y) a^{+}(x)(a(x)+a(y)) d x d y} \\
{\left[a_{0},\left[a_{0},\left[H, a_{0}^{+}\right]\right]\right]=2 V^{-2} \int_{\Lambda} \phi(x-y) d x d y a_{0} .} \\
{\left[a_{0},\left[a_{0},\left[\left[H, a_{0}^{+}\right], a_{0}^{+}\right]\right]\right]=2 V^{-2} \int_{\Lambda} \phi(x-y) d x d y .}
\end{gathered}
$$

\section{References}

1. Bogoliubov, N. N.: J. Phys. (USSR) II, 23 (1947).

2. Huang, K.: Phys. Rev. 115, 765 (1959).

3. Ezawa, H.: J. Math. Phys. 6, 380 (1965).

4. -, and M. Luban: J. Math. Phys. 8, 1285 (1967).

5. Bogoliubov, N. N.: Phys. Abh., S.U. 6, 1, 229 (1962).

6. HAAG, R.: Nuovo Cimento 25, 287 (1962).

7. Ruelle, D.: Helv. Phys. Acta 36, 789 (1963).

8. Fisher, M.: Arch. Ratl. Mech. Anal. 17, 377 (1964).

9. Bogoliubov, N. N. JR.: Physica 32, 933 (1966).

10. RUELLE, D.: In: Lectures in theoretical physics. Vol. 6. Colorado: University of Colorado Press 1964.

11. - Helv. Phys. Acta 36, 183 (1963).

12. Fisher, M., and D. Ruelde: J. Math. Phys. 7, 260 (1966).

13. Riesz, F., and B. Sz. NAGY: Functional analysis. New York: Ungar 1955.

14. WeyL, H.: Proc. Natl. Acad. Sci. 35, 408 (1949).

15. Trotter, H.: Proc. Am. Math. Soc. 10, 545 (1959).

16. HILLE, E., and R. S. PHmLIPS: Functional analysis and semi-groups. Am. Math. Soc. Colloquium Pub., Vol. 31 (1957).

17. Huang, K.: Statistical mechanics. New York: Wiley 1963.

18. Glauber, R. J.: Phys. Rev. 131, 2766 (1963).

19. LeE, T. D., and C. N. YANG: Phys. Rev. 117, 897 (1960).

20. Araki, H., and E. J. Woods: J. Math. Phys. 4, 637 (1963).

21. Lee, T. D., and C. N. YANG: Phys. Rev. 113, 1165 (1959).

22. Dele'Antonio, G. F.: Lecture notes. Brandeis 1966.

Dr. J. GINIBRE

Department of Physics

New York University

Mercer Street

New York, USA 